\title{
A NOTE ON AN ASYMMETRIC MIXED BOUNDARY VALUE PROBLEM FOR A HALF SPACE WITH A CYLINDRICAL CAVITY
}

\author{
by PREM NARAIN
}

(Received 6 August, 1964)

In recent years interest in the mixed boundary value problems of mathematical physics has increased appreciably because of various applications. The mixed boundary value problems for simply-connected regions have been investigated widely and it can reasonably be hoped that within a short time the theory will reach a satisfactory stage. It appears, however, that very few problems for multiply-connected domains have been solved. Recently Srivastav [2] has considered the problem of finding an axisymmetric potential function for a half space with a cylindrical cavity subject to mixed type boundary conditions. In a subsequent paper [1], Srivastav extends the analysis to the asymmetric problem and formulates the problem in terms of dual integral equations involving Bessel functions of the first and second kinds whose solution leads to the solution of the potential problem. The latter paper, however, involves heavy manipulations and complicated contour integrals.

In this note, making use of the technique adopted by Sneddon [3] for solving the problem of an electrified disk inside an earthed cylinder, we show that the integral equation obtained by Srivastav [2] can be obtained in a straightforward manner. The analysis used is purely formal and no conditions have been determined under which changes of order of integration and so on are legitimate.

Two types of boundary value problems of interest arise.

(a) The lateral surface of the cylindrical cavity is maintained at zero potential.

(b) The normal derivative of the electrostatic potential across the lateral surface of the cavity is zero.

In this note we shall consider the problem (a), which can be stated as follows:

Determine a function $\phi(\rho, \theta, z)$ which satisfies Laplace's equation

$$
\nabla^{2} \phi=\frac{\partial^{2} \phi}{\partial \rho^{2}}+\frac{1}{\rho} \frac{\partial \phi}{\partial \rho}+\frac{1}{\rho^{2}} \frac{\partial^{2} \phi}{\partial \theta^{2}}+\frac{\partial^{2} \phi}{\partial z^{2}}=0 \quad(1<\rho<\infty, 0 \leqq \theta \leqq 2 \pi, 0 \leqq z<\infty)
$$

and the boundary conditions

$$
\begin{aligned}
\phi(\rho, \theta, z) \rightarrow 0 & \text { as } \sqrt{ }\left(\rho^{2}+z^{2}\right) \rightarrow \infty, \\
\phi(1, \theta, z)=0 & (0 \leqq \theta \leqq 2 \pi, 0 \leqq z<\infty), \\
\phi(\rho, \theta, 0)=0 & (0 \leqq \theta \leqq 2 \pi, 1 \leqq \rho<a), \\
\frac{\partial}{\partial z} \phi(\rho, \theta, 0)= & -\left(f_{n}(\rho) \cos n \theta+g_{n}(\rho) \sin n \theta\right) \quad(0 \leqq \theta \leqq 2 \pi, a<\rho<\infty) .
\end{aligned}
$$


We shall assume that $g(\rho) \equiv 0$ for the sake of convenience in presentation. In case $g(\rho) \neq 0$, the modification in the method is obvious.

A suitable representation for the harmonic function $\phi(\rho, \theta, z)$ which tends to zero as $\sqrt{ }\left(\rho^{2}+z^{2}\right) \rightarrow \infty$ is given by the equation

$$
\phi(\rho, \theta, z)=\cos n \theta\left[\int_{0}^{\infty} \psi_{n}(\xi) J_{n}(\xi \rho) e^{-\xi z} d \xi+\int_{0}^{\infty} \chi_{n}(\xi) K_{n}(\xi \rho) \sin (\xi z) d \xi\right],
$$

where $J_{n}$ is the Bessel function of the first kind and $K_{n}$ is the modified Bessel function of the second kind. The boundary conditions (3), (4) and (5) are fulfilled if we can determine $\psi_{n}(\xi)$ and $\chi_{n}(\xi)$ such that following three equations are satisfied:

$$
\begin{aligned}
& \int_{0}^{\infty} \psi_{n}(\xi) J_{n}(\xi) e^{-\xi z} d z+\int_{0}^{\infty} \chi_{n}(\xi) \sin (\xi z) K_{n}(\xi) d \xi=0 \quad(0<z<\infty), \\
& \int_{0}^{\infty} \psi_{n}(\xi) J_{n}(\xi \rho) d \xi=0 \quad(1<\rho<a), \\
& \int_{0}^{\infty} \xi \psi_{n}(\xi) J_{n}(\xi \rho) d \xi=f_{n}(\rho)+\int_{0}^{\infty} \xi \chi_{n}(\xi) K_{n}(\xi \rho) d \xi \quad(\rho>a) .
\end{aligned}
$$

From the theory of Fourier transforms it easily follows that the equation (7) is equivalent to the equation

$$
K_{n}(\xi) \chi_{n}(\xi)+\frac{2 \xi}{\pi} \int_{0}^{\infty} \frac{\psi_{n}(t) J_{n}(t)}{\left(t^{2}+\xi^{2}\right)} d t=0
$$

Let us suppose now that

$$
\int_{0}^{\infty} \psi_{n}(\xi) J_{n}(\xi \rho) d \xi=\left\{\begin{array}{cl}
0 & (1<\rho<a), \\
\rho^{-n} \int_{a}^{\rho} \frac{g(t)}{\sqrt{\left(\rho^{2}-t^{2}\right)}} d t & (a<\rho<\infty),
\end{array}\right.
$$

where $g(t)$ is an as yet unspecified function. On using the inversion theorem for Hankel transforms and then interchanging the order of integration, we obtain

$$
\frac{\psi_{n}(\xi)}{\xi}=\int_{a}^{\infty} g(t) \int_{t}^{\infty} \frac{\rho^{1-n} J_{n}(\xi \rho)}{\sqrt{\left(\rho^{2}-t^{2}\right)}} d \rho d t
$$

Since $[4,(32)$, p. 25$]$

$$
\int_{t}^{\infty} \frac{\rho^{1-n} J_{n}(\xi \rho)}{\sqrt{\left(\rho^{2}-t^{2}\right)}} d \rho=\sqrt{\frac{\pi}{2 \xi}} t^{\frac{1}{2}-n} J_{n-\frac{1}{2}}(t \xi)
$$

we therefore have

$$
\psi_{n}(\xi)=\sqrt{\frac{\pi \xi}{2}} \int_{a}^{\infty} g(t) t^{\frac{1}{2}-n} J_{n-\frac{1}{2}}(t \xi) d t
$$


If we put the above value of $\psi_{n}(\xi)$ in the equation (8) and interchange the order of integration, we find that (8) is identically satisfied by $\psi_{n}(\xi)$ for all those functions $g(t)$ for which we can interchange the order of integration. If we substitute the value of $\psi_{n}(\xi)$ from (12) in the equation (10), it is reduced to the equation

$$
\chi_{n}(\xi)+\sqrt{\frac{2 \xi}{\pi}} \frac{I_{n}(\xi)}{K_{n}(\xi)} \int_{a}^{\infty} \frac{g(u)}{u^{n-\frac{1}{2}}} K_{n-\frac{1}{2}}(u \xi) d u .
$$

Multiplying (9) by $\rho^{1-n}$ and integrating over $(\rho, \infty)$ with respect to $\rho$, we find that

$$
\int_{0}^{\infty} \psi_{n}(\xi) J_{n-1}(\xi \rho) d \xi=\rho^{n-1} \int_{\rho}^{\infty} \rho^{1-n} f_{n}(\rho) d \rho+\int_{0}^{\infty} \chi_{n}(\xi) K_{n}(\xi \rho) d \xi
$$

If we substitute the value of $\psi_{n}(\xi)$ from (12) in the equation (14), we find that it is reduced to the equation

$$
\int_{\rho}^{\infty} \frac{g(t) t^{1-2 n}}{\sqrt{\left(t^{2}-\rho^{2}\right)}} d t=\int_{\rho}^{\infty} \rho^{1-n} f_{n}(\rho) d \rho+\rho^{1-n} \int_{0}^{\infty} \chi_{n}(\xi) K_{n-1}(\xi \rho) d \xi,
$$

which is easily shown to be equivalent to the equation

$$
\begin{aligned}
g(t) t^{1-2 n}= & -\frac{2}{\pi} \frac{d}{d t} \int_{1}^{\infty} \frac{\rho}{\sqrt{\left(\rho^{2}-t^{2}\right)}}\left\{\int_{\rho}^{\infty} u^{1-n} f_{n}(u) d u\right\} d \rho \\
& -\frac{2}{\pi} \frac{d}{d t} \int_{t}^{\infty}-\frac{\rho^{2-n}}{\sqrt{ }\left(\rho^{2}-t^{2}\right)}\left\{\int_{0}^{\infty} \chi_{n}(\xi) K_{n-1}(\xi \rho) d \xi\right\} d \rho .
\end{aligned}
$$

The expression for $g(t)$ still involves an unknown $\chi_{n}(\xi)$. If we now combine (13) and (15) we obtain the integral equation

where

$$
g(t) t^{1-2 n}=-\frac{2}{\pi} \frac{d}{d t} \int_{t}^{\infty} \frac{\rho}{\sqrt{\left(\rho^{2}-t^{2}\right)}}\left\{\int_{\rho}^{\infty} u^{1-n} f_{n}(u) d u\right\} d \rho-\frac{2}{\pi} t^{t-n} \int_{a}^{\infty} \frac{g(u)}{u^{n-\frac{1}{2}}} K(u \cdot t) d u,
$$

$$
K(u, t)=\int_{0}^{\infty} \xi \frac{I_{n}(\xi)}{K_{n}(\xi)} K_{n-\frac{t}{2}}(u \xi) K_{t-n}(t \xi) d \xi,
$$

which is the same as that obtained by Srivastav [1].

I am greatly indebted to Dr R. P. Srivastav for many helpful discussions in connection with the problem discussed in this paper.

\section{REFERENCES}

1. R. P. Srivastav, A pair of dual integral equations involving Bessel functions of first kind and second kind, Proc. Edinburgh Math. Soc. (to be published).

2. R. P. Srivastav, An axisymmetric mixed boundary value problem for a half-space with a cylindrical cavity, J. Math. Mech. 13 (1964), 385-393.

3. I. N. Sneddon, A note on an electrified disk inside an earthed cylinder, Proc. Cambridge Philos. Soc. 58 (1962), 621-624.

4. A. Erdélyi et al., Tables of integral transforms, Vol. II (New York, 1964).

INDIAN INSTITUTE OF TECHNOLOGY

\section{KANPUR}

INDLA 